\title{
An Introduction to the Book Titled Nature of
}

\section{Computation and Communication}

\author{
Phan Cong Vinh ${ }^{1}$ \\ ${ }^{1}$ Nguyen Tat Thanh University, Ho Chi Minh City, Vietnam
}

Abstract

We introduce the book with the title of "Nature of Computation and Communication" edited by Prof. Phan Cong Vinh, Dr. Emil Vassev and Prof Mike Hinchey, and published by Springer in 2015 to scientists, researchers, professionals and students in the field of computing. Hopefully, they will find this book provoking for their research related to hot topics of computing and being useful to their future work.

Received on 12 May 2015; accepted on 12 May 2015; published on 04 August 2015

Keywords: Collective adaptive system, Formal methods, Self-adaptive system

Copyright (C) 2015 Phan Cong Vinh, licensed to ICST. This is an open access article distributed under the terms of the Creative Commons Attribution license (http://creativecommons.org/licenses/by/3.0/), which permits unlimited use, distribution and reproduction in any medium so long as the original work is properly cited.

doi:10.4108/eai.4-8-2015.150045

\section{Introduction}

The 1st International Conference on naTure of Computation and Communication (ICTCC 2014) was a place for highly original ideas about how the nature is going to shape computing systems of the future. There, it focused on rigorous approaches and cuttingedge solutions, which were to make a formal basis more accessible to researchers, scientists, professionals and students as well as developers and practitioners in ICT by providing them with state-of-the-art research results, applications, opportunities and future trends.

The ICTCC 2014 was an international scientific conference research in the fields related to nature of computation and communication held during November 24-25, 2014 in Ho Chi Minh City of Vietnam. This conference provided an excellent opportunity for researchers to discuss natural approaches and techniques for computation and communication. The proceedings of ICTCC 2014 [1] were published by Springer in series Lecture Notes of the Institute for Computer Sciences, Social Informatics and Telecommunications Engineering (LNICST) (indexed by DBLP, EI, Google Scholar, Scopus, Thomson ISI).

\footnotetext{
${ }^{*}$ Corresponding author. Email: pcvinh@ntt.edu.vn
}

For this edition, the Program Committee received over hundred submissions from twenty countries and each paper was reviewed by at least three expert reviewers. Thirty four papers were selected after intensive discussions held among the Program Committee members. The excellent reviews and lively discussions of the Program Committee members and external reviewers were highly appreciated in the review process.

This book, with papers contributed by researchers from academia and industry, serves as a technical guide and reference material for researchers, scientists, professionals and students in computer science and computer engineering as well as developers and practitioners in computing and networking systems design by providing them with state-of-the-art research findings and future opportunities and trends. These contributions include formal approaches and applications in natural computing. In particular, the book covers existing and emerging research issues in the formal methods for natural computing.

\section{Contents of the Book}

The book has thirty four papers organized into two sections. First section contains five papers addressing formal and practical aspects of self-adaptive systems. 


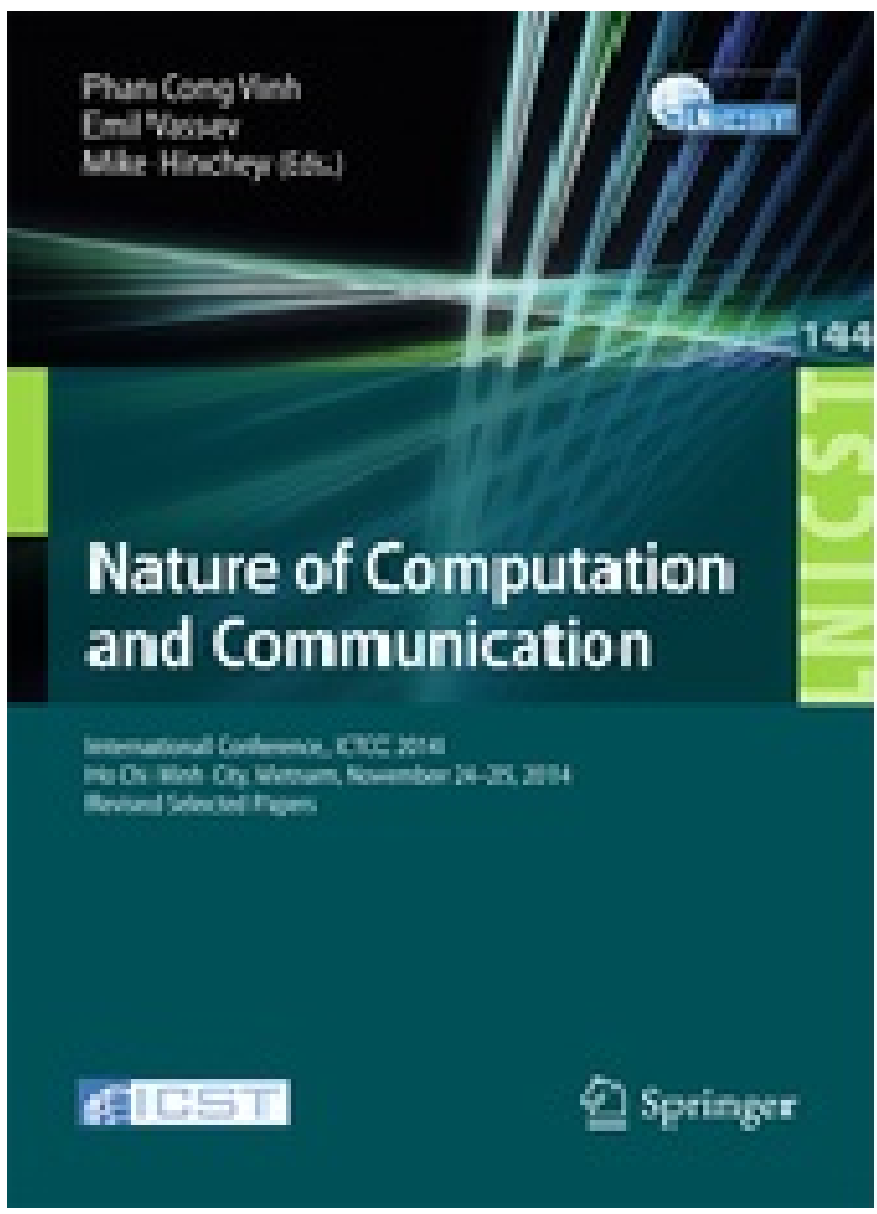

Figure 1. Cover of the book

Second section consists of twenty nine papers presenting formal and practical aspects of collective adaptation and others.

\subsection{Formal Methods for Self-Adaptive Systems}

A self-adaptive system changes its behavior in response to stimuli from its execution and operational environment. As software is used for more pervasive and critical applications, support for self-adaptation is increasingly seen as vital in avoiding costly disruptions for repair, maintenance and evolution of systems. Selfadaptation may result in changes to some functionality, algorithms, or system parameters, as well as to the system's structure or any other system aspect. Moreover, an autonomic self-adaptive system may have intrinsic intelligence that may help it reason about situations where autonomous decision making is required. The major challenges to overcome in order to achieve adequate self-adaptive behavior are related to monitoring, quality and quantity of knowledge and awareness. A successful self-adaptive system meets those challenges to emphasize features like adaptability, autonomy, mobility, robustness, and dynamicity.
Developing self-adaptive systems requires new approaches that can efficiently tackle the problems of expressing autonomy requirements, designing and implementing self-adaptive features, and efficiently testing self-adaptive behavior. This section of the book contains both theoretical and applied contributions related to formal methods in engineering self-adaptive systems as follows:

- Modular Design and Verification of Distributed Adaptive Real-Time Systems

- Modeling Swarm Robotics with KnowLang

- Reasoning on Data Streams: An Approach to Adaptation in Pervasive Systems

- Autonomic Computing Software for Autonomous Space Vehicles

- Logic-Based Modeling of Information Transfer in Cyber-Physical Multi-Agent Systems

\subsection{Nature of Computation and Communication}

The socio-technical structure of our community increasingly depends on systems, which are built as a collection of varied agents and are tightly coupled with humans and social interrelations. Their agents more and more need to be able to develop, cooperate and work all by themselves as a part of an artificial community. Hence, for such collective adaptive systems (CASs), one of major challenges is how to support self-adaptation in the face of changing interactions. In other words, how does a CAS understand relevant interrelations and then self-adapt to become better able to live in its interactions? Dealing with this grand challenge of CASs requires a well-founded modeling and in-depth analysis on the notions of self-adaptation and self-adaptive trait. With this aim, this section of the book covers some highlighted contents as follows.

- Categorical structures of self-adaptation in CASs where a collection of entities is able to selfadapt its configuration and self-optimize its performance in the face of changing interactions.

- Self-adaptation monoid to shape series of selfadaptive traits in CASs.

- Justifying equivalence between two series of selfadaptive traits based on a powerful method socalled proof principle of coinduction

\section{Remarkable Features of the Book}

This book has the following remarkable features:

- Provides a comprehensive reference on natural aspects of computation and communication. 
- Presents state-of-the-art natural aspects of computation and communication.

- Formally specifies, develops and verifies natural aspects of computation and communication.

- Includes illustrative figures facilitating easy reading.

- Discusses emerging trends and open research problems on nature of computation and communication.

The book serves as a comprehensive and essential reference on natural aspects of computation and communication and is intended as a collection of revised selected papers for senior undergraduate and graduate-level courses. It can also be used as a supplementary book for graduate courses. The book is a useful resource for the students and researchers to learn natural aspects of computation and communication. In addition, it will be valuable to professionals from both the academia and industry and generally serves instant appeal to the people who would like to contribute to natural computing technologies.

\section{References}

[1] P.C. Vinh, E. Vassev, M. Hinchey(2015) Nature of Computation and Communication, Springer, 1st ed. 